Research Paper

\title{
The efficacy and safety of long- versus short-interval transarterial chemoembolization in unresectable hepatocellular carcinoma
}

\author{
Zhi-Wen Yang1,2*, Wei He1,2* Yun Zheng1,2, Ru-Hai Zou1,3, Wen-Wu Liu1,2, Yuan-Ping Zhang1,2, Chen-Wei \\ Wang1,2, Yong-Jin Wang ${ }^{1,2}$, Yi-Chuan Yuan ${ }^{1,2}$, Bin-Kui Li ${ }^{1,2}{ }^{\bowtie}$, Yun-Fei Yuan ${ }^{1,2}$ \\ 1. State Key Laboratory of Oncology in South China, Sun Yat-Sen University Cancer Center, Guangzhou, China \\ 2. Department of Hepatobiliary Oncology, Sun Yat-Sen University Cancer Center, Guangzhou, China \\ 3. Department of Ultrasound, Sun Yat-Sen University Cancer Center, Guangzhou, China \\ * These authors contributed equally to this work. \\ $\bowtie$ Corresponding authors: Yunfei Yuan, E-mail: yuanyf@mail.sysu.edu.cn or Binkui Li, E-mail: libinkui@mail.sysu.edu.cn \\ (C) Ivyspring International Publisher. This is an open access article distributed under the terms of the Creative Commons Attribution (CC BY-NC) license \\ (https://creativecommons.org/licenses/by-nc/4.0/). See http://ivyspring.com/terms for full terms and conditions.
}

Received: 2017.12.07; Accepted: 2018.06.16; Published: 2018.10.16

\begin{abstract}
Background: To compare the efficacy and safety of long- versus short-interval of transarterial chemoembolization (TACE) in unresectable hepatocellular carcinoma (HCC) patients.

Methods: This retrospective analysis enrolled 574 patients with unresectable HCC who underwent at least two sessions of TACE between January 2007 and December 2014. The patients were divided into a short-interval group (SIG) and a long-interval group (LIG) based on the median TACE interval of the first two sessions. Propensity score matching (PSM) identified 476 patients for a comparison of overall survival (OS) and safety.

Results: Before matching, the LIG had a longer OS than the SIG (Median: 12.1 vs. 8.7 months; $P=0.003$ ). After matching, median OS in the SIG and LIG were 9.1 and 14.2 months $(P<0.001)$. The 1-, 2-, and 3 -year survival rates were $37.5 \%, 17.1 \%$, and $9.9 \%$ for SIG and $50.1 \%, 19.3 \%$, and $11.6 \%$ for LIG, respectively. The TACE interval was an independent prognostic factor for OS. The LIG had a longer OS than the SIG in Barcelona Clinic liver cancer (BCLC) stage $C$ patients (Median: 10.2 vs. 5.8 months; $P<$ 0.001 ), but not in BCLC-A or B. The postoperative adverse rates were similar in matched SIG and LIG patients $(29.4 \%$ vs. $33.6 \%, P=0.324)$.
\end{abstract}

Conclusions: A long interval between the first two sessions of TACE resulted in a better OS than a short interval in patients with unresectable BCLC C-stage HCC.

Key words: unresectable hepatocellular carcinoma; transarterial chemoembolization; interval

\section{Introduction}

Liver cancer is the third leading cause of cancer death worldwide[1]. Approximately $70 \%$ of HCC patients are diagnosed at an intermediate or advanced stage when curative treatments (including hepatic resection, ablation, and liver transplantation) are no longer feasible.

Transarterial chemoembolization (TACE) is widely recommended in clinical practice due to its high efficacy in patients with unresectable HCC[2-7]. TACE can significantly increase the 2-year survival rate from $27 \%$ to $63 \%$ compared with systemic therapy and supportive care in patients with unresectable HCC $[8,9]$. There are increasing data to support that repeated TACE can significantly improve the tumor response rate and prolong overall survival (OS)[10, 11]. However, TACE is also associated with increased adverse effects and liver damage, potentially preventing a better prognosis[12-16]. Currently, the interval for repeated TACE varies among different medical centers, ranging from one to three months[17-20]. However, the efficacy and safety of various intervals of repeated 
TACE remain unknown.

We aimed to retrospectively compare the outcomes of TACE treatment with a long interval and a short interval between the first and second sessions in patients with unresectable HCC. A propensity score matching (PSM) analysis was used to reduce any potential confounding bias at baseline.

\section{Methods}

\section{Patients}

A total of 1443 patients with unresectable HCC received TACE as an initial treatment at the our Center between January 2007 and December 2014. The diagnosis of HCC was confirmed by radiological examination based on the European Association for the Study of Liver (EASL) criteria[21]. The interval between the first two TACE sessions was defined as the duration from the date of the first TACE procedure to the date of the second TACE procedure. We collected the following parameters as possible predictors of survival: demographics, risk factors of HCC and clinical data. Liver functional reserve was assessed using Child-Pugh grading and albumin-bilirubin (ALBI) grades.

The following inclusion criteria were used: (a) TACE as the primary treatment for HCC; (b) at least two TACE treatments received; (c) no intent for subsequent curative treatment including hepatic resection, ablation, or liver transplantation; (d) age between 18 and 75 years; (e) Child-Pugh A or B liver function; and ( $\mathrm{f}$ ) an Eastern Cooperative Oncology Group performance status of $0-2$. The following exclusion criteria were used: (a) a repeated TACE interval of more than 90 days or less than 21 days; (b) the preoperative presence of severe conditions, such as cardiovascular disease or renal insufficiency; or (c) diagnosis of another malignancy. Patients with TACE intervals greater than the median interval were grouped into the short-interval group (SIG), and the remaining patients were grouped into the long-interval group (LIG).

The study protocol conformed to the ethical guidelines of the 1975 Declaration of Helsinki, and the Ethics Committee of the Sun Yat-Sen University Cancer Center approved this study. Written informed consent was obtained before treatment.

\section{TACE procedure}

TACE was performed as previously described[22]. In all cases, the TACE procedure was performed under local anesthesia by a hepatologist with more than 10 years of experience in interventional therapy for HCC. First, visceral angiography was routinely performed to assess tumor vascularity, vascular anatomy and portal vein patency using a 5-F catheter. We excluded the existence of an arteriovenous shunt based on visceral angiography. Then, depending on the tumor distribution, tumor size and arterial supply to the tumor, the tip of the catheter was advanced into the right or left hepatic artery to perform selective arterial embolization. A 2.9-F microcatheter (Terumo, Tokyo, Japan) was used if the 5-F catheter could not advance into the tumor-feeding artery. Next, $300 \mathrm{mg}$ of carboplatin (Bristol Myers Squibb, New York, NY) was used for hepatic artery infusion chemotherapy. Then, a mixture of $50 \mathrm{mg}$ of epirubicin (Pharmorubicin; Pfizer, Wuxi, China), $8 \mathrm{mg}$ of mitomycin C (Zhejiang Hisun Pharmaceutical, Taizhou, China) or $500 \mathrm{mg}$ of 5-FU, and 2-5 ml of lipiodol (Lipiodol Ultra-Fluide; André Guerbet Laboratories, Aulnay Sous-Bois, France) was injected into the tumor distribution for chemolipiodolization. Pure lipiodol was injected into the arterial supply of the tumor until a substantial reduction in flow to the tumor distribution was achieved. Finally, X-ray imaging of the chest and abdomen was performed to verify the distribution of lipiodol and to exclude ectopic embolization.

\section{Follow-up and repeat TACE}

Enhanced computed tomography (CT) or magnetic resonance imaging (MRI) was performed one week before and four to six weeks after TACE-2 by experienced radiologists and hepatologists for an assessment of the radiologic tumor response according to the Modified Response Evaluation Criteria in Solid Tumors criterion (mRECIST)[23]. Detailed blood tests and chest radiography were performed during follow-up visits. The decision for repeated TACE was made by a multidisciplinary team of experts including a radiologist, interventional radiologists, oncologists and surgeons. Generally, repeated TACE treatment was performed at 4- to 8 -week intervals until the following end points were reached: (1) tumor progression; (2) deterioration of liver function; (3) Eastern Cooperative Group score > 2; or (4) patients' refusal to TACE.

TACE-related adverse events were evaluated within four weeks after treatment. Hematotoxicity was evaluated and graded according to the common terminology criteria for adverse events (CTCAE) version 4.0 [24], and the intensity was characterized as mild, moderate, severe, life-threatening, or fatal. Hyperpyrexia was defined as the presentation of a fever over 39 degrees within one week after TACE-2.

\section{Statistical analysis}

We performed PSM analysis to reduce the bias of variables associated with treatment selection and outcome prediction. Variables identified by Logistic 
regression and were considered as important based on clinical practice were introduced to generate a propensity score. A one-to-one nearest neighbor matching algorithm with a caliper of 0.2 and without replacement was used.

We applied an independent sample t-test and the Mann-Whitney U-test to analyze continuous variables. The chi-square test and the Kruskal-Wallis test were used to analyze binary and ordinal categorical variables, respectively. The OS was calculated as the interval between the date of initial TACE and death or the last follow-up. We used the Kaplan-Meier method and the log-rank test to estimate and compare OS. Median survival times and their $95 \%$ confidence intervals $(\mathrm{CI})$ were reported. A univariate analysis was performed to identify the factors that predicted survival. All variables with $\mathrm{P}<$ 0.05 based on the univariate analysis were included in a multivariate analysis. The multivariate analysis was performed with the Cox proportional hazards model. All statistical analyses were performed by SPSS (IBM SPSS Statistics for WINDOWS, Version 20.0, IBM Corp., Armonk, NY, USA) and PSM for SPSS, Version 3.02 (Felix Thoemmes, Cornell University/University of Tübingen, and Wang Liao, Cornell University). The P-values are the results of two-sided tests. A level of 0.05 was defined for statistically significant differences.

\section{Results}

\section{Study population}

A total of 1443 HCC patients initially treated with TACE between January 2007 and December 2014 at the Sun Yat-Sen University Cancer Center were included in the study. Of these, 869 individuals were excluded for the following reasons: only one session of TACE $(n=203)$, subsequent hepatic resection $(n=$ $223)$, ablation $(n=188)$, or liver transplantation $(n=2)$, an interval of more than 90 days ( $\mathrm{n}=171$ patients) or less than 21 days $(n=61)$ between the first two TACE sessions, a history of other malignant tumors $(n=5)$ and lack of follow-up $(n=16)$. Ultimately, 574 patients were enrolled (Figure 1). Among them, the numbers of patients who underwent $2,3,4$, or 5 or more sessions of TACE were 378 (65.9\%), $123(21.4 \%), 47$ $(8.2 \%)$, and $26(4.5 \%)$, respectively. The median interval between the first two TACE sessions was 48 days.

The clinical characteristics observed before and after patient matching are shown in Table 1, Table 2. Patients with intervals shorter than the median interval were assigned to the SIG $(n=315)$, and the remaining patients were assigned to the LIG $(n=259)$. The numbers of patients with ALBI grades of $-1,-2$, and -3 prior to initial TACE were $170(54.0 \%), 142$ $(45.1 \%)$, and $3(1.0 \%)$, respectively, for SIG patients and $142(54.8 \%), 115$ (44.4\%), and 2 (0.8\%), respectively, for LIG patients. The distributions of ALB were similar in the SIG and LIG before and after matching (Table 1, 2; $\mathrm{P}=0.824,0.960$ ).

The SIG had more male patients $(94.0 \%$ vs. $89.6 \%, \mathrm{P}=0.038)$, larger tumor sizes $(\mathrm{cm}, 10.4$ vs. 9.4, $\mathrm{P}$ $=0.006)$, higher $\mathrm{\gamma}$-glutamyl transferase levels $(\mathrm{U} / \mathrm{L}$, median level: 190.6 vs. $151.6, \mathrm{P}=0.003)$, and higher white blood cell counts $\left(10^{12} / \mathrm{L}\right.$, median level: $7.00 \mathrm{vs.}$ $6.60, \mathrm{P}=0.034)$ than the LIG.

\section{Overall survival}

The mean length of follow-up was 11.0 months (range, 1.0 - 124.0 months). The OS was greater in the LIG than in the SIG $(\mathrm{P}=0.003$; hazard ratio [HR], 0.752 ; $95 \%$ CI, 0.622 to 0.908 ; Figure 2). The median survivals of the SIG and LIG were 8.7 and 12.1 months $(P=0.003)$, respectively. The 1-, 2-, and 3-year survival rates were $36.3 \%, 17.6 \%$, and $10.3 \%$, respectively, in the SIG and $50.7 \%, 20.7 \%$, and $11.4 \%$ in the LIG, respectively.

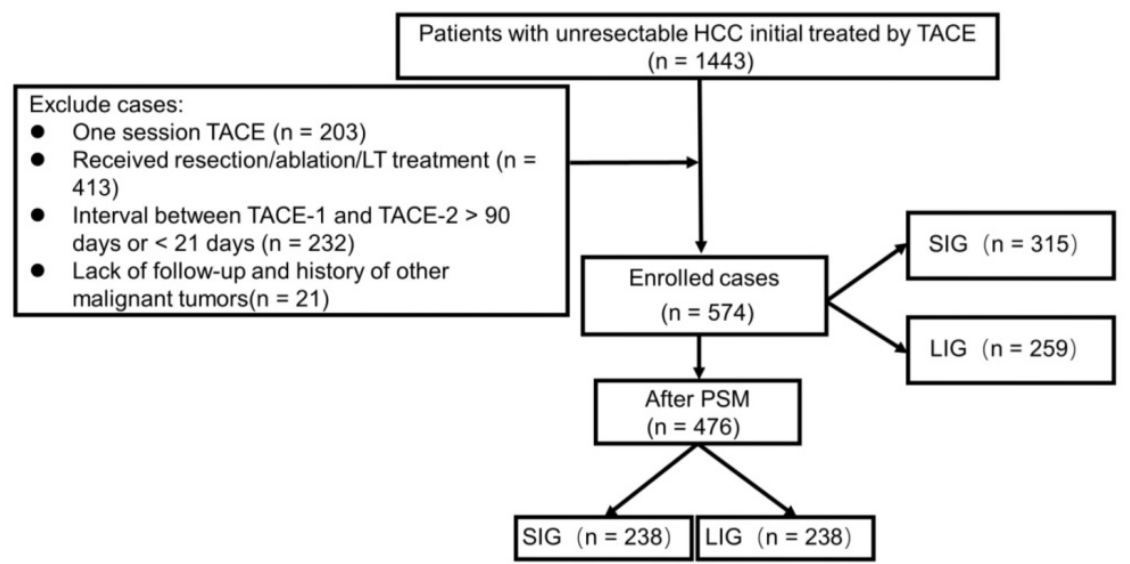

Figure 1. Flow chart of the study: Patient selection and final study population. SIG: Short interval group, 21-48 days interval; LIG: Long interval group, 49-90 days interval; PSM: Propensity score matching 
Table 1. Characteristics of all patients before first session TACE

\begin{tabular}{|c|c|c|c|c|}
\hline \multirow[b]{2}{*}{ Variables } & & \multicolumn{2}{|c|}{ All patients } & \multirow[t]{2}{*}{$\mathrm{p}$} \\
\hline & & Short-interval $(n=315)$ & Long-interval $(n=259)$ & \\
\hline Gender ( M/F ) & & $296 / 19$ & $232 / 27$ & 0.038 \\
\hline Age, year & & $51(25-82)$ & $53(18-80)$ & 0.146 \\
\hline Hepatitis B ( \% ) & & $280(88.89 \%)$ & $220(84.94 \%)$ & 0.160 \\
\hline Hepatitis C ( \% ) & & $3(0.95 \%)$ & $8(3.09 \%)$ & 0.073 \\
\hline $\mathrm{WBC}, 10^{9} / \mathrm{L}$ & & $7.00(2.70-17.60)$ & $6.60(2.16-24.60)$ & 0.034 \\
\hline $\mathrm{RBC}, 10^{9} / \mathrm{L}$ & & $4.64(2.76-7.35)$ & $4.61(2.50-7.34)$ & 0.542 \\
\hline $\mathrm{HB}, \mathrm{g} / \mathrm{L}$ & & $138.00(83.70-198.00)$ & $137.00(65.40-185.00)$ & 0.146 \\
\hline PLT, $10^{9} / \mathrm{L}$ & & $201.4(31.00-749.70)$ & $185.00(12.10-604.00)$ & 0.090 \\
\hline ALT, U/L & & $47.10(8.70-322.70)$ & $46.40(9.10-455.00)$ & 0.533 \\
\hline AST, U/L & & $62.40(17.00-736.60)$ & $60.50(9.70-1332.50)$ & 0.285 \\
\hline GGT, U/L & & $190.60(24.50-2480.00)$ & $151.60(20.70-1524.10)$ & 0.003 \\
\hline $\mathrm{ALB}, \mathrm{g} / \mathrm{L}$ & & $40.20(26.70-73.21)$ & $40.00(22.30-54.00)$ & 0.994 \\
\hline TBIL, $\mu \mathrm{mol} / \mathrm{L}$ & & $15.40(3.60-225.00)$ & $14.70(5.60-616.30)$ & 0.180 \\
\hline Creatine, $\mu \mathrm{mol} / \mathrm{L}$ & & $73.16(36.5-217.40)$ & $73.30(3.35-112.90)$ & 0.960 \\
\hline PT, sec & & $12.20(8.70-23.00)$ & $12.30(8.70-20.30)$ & 0.297 \\
\hline $\mathrm{CRP}, \mathrm{mg} / \mathrm{L}$ & & $11.83(0.25-208.18)$ & $10.65(0.21-241.30)$ & 0.078 \\
\hline INR & & $1.06(0.76-2.05)$ & $1.06(0.76-1.61)$ & 0.390 \\
\hline \multirow[t]{3}{*}{$\mathrm{AFP}, \mathrm{ng} / \mathrm{ml}$} & $<200$ & $107(33.97 \%)$ & $104(40.15 \%)$ & \\
\hline & $200-400$ & $13(4.13 \%)$ & $11(4.25 \%)$ & \\
\hline & $>400$ & $195(61.90 \%)$ & $144(55.60 \%)$ & 0.119 \\
\hline Tumor-Size, cm & & $10.4(1.0-19.8)$ & $9.4(1.0-25.0)$ & 0.006 \\
\hline \multirow[t]{2}{*}{ Tumor number } & Solitary & $121(38.41 \%)$ & $83(32.05 \%)$ & \\
\hline & Multifocal & $194(61.59 \%)$ & $176(67.95 \%)$ & 0.113 \\
\hline \multirow[t]{2}{*}{ PVTT } & Absent & $204(64.76 \%)$ & $181(69.88 \%)$ & \\
\hline & Present & $111(35.24 \%)$ & $78(30.12 \%)$ & 0.194 \\
\hline \multirow[t]{3}{*}{ Times of TACE } & 2 & $202(64.13 \%)$ & $176(67.95 \%)$ & \\
\hline & 3 & $69(21.90 \%)$ & $54(20.85 \%)$ & \\
\hline & $>=4$ & $44(13.97 \%)$ & $29(11.20 \%)$ & 0.291 \\
\hline \multirow[t]{2}{*}{ Child-Pugh stage } & A & $295(93.64 \%)$ & $241(93.05 \%)$ & \\
\hline & B & $20(6.36 \%)$ & $18(6.95 \%)$ & 0.773 \\
\hline \multirow[t]{3}{*}{ BCLC stage } & A & $75(23.80 \%)$ & $59(22.78 \%)$ & \\
\hline & B & $129(40.96 \%)$ & $121(46.72 \%)$ & \\
\hline & $\mathrm{C}$ & $111(35.24 \%)$ & $79(30.50 \%)$ & 0.520 \\
\hline \multirow[t]{2}{*}{ ABIL grade } & 1 & $170(53.97 \%)$ & $142(54.83 \%)$ & \\
\hline & 2 & $142(45.08 \%)$ & $115(44.40 \%)$ & \\
\hline \multirow[t]{2}{*}{ Iodized oil, ml } & 3 & $3(0.95 \%)$ & $2(0.77 \%)$ & 0.824 \\
\hline & & $19 \pm 7.5$ & $18.0 \pm 7.2$ & 0.173 \\
\hline
\end{tabular}

Abbreviations: WBC, white blood cell; RBC, red blood cell; HB, hemoglobin; PLT, platelet; ALT, alanine transaminase; AST, aspartate aminotransferase; GGT,

gamma-glutamyl transpeptidase; ALB, albumin; TBIL, total bilirubin; PT, prothrombin time; CRP, C-reactive protein; INR, international normalized ratio; AFP, alpha

fetoprotein; PVTT, portal vein tumor thrombus; TACE, transcatheter arterial chemoembolization; BCLC, Barcelona Clinical Liver Cancer; ABIL, Albumin-Bilirubin.

Table 2. Characteristics of after matching patients before first session TACE

\begin{tabular}{|c|c|c|c|c|}
\hline \multirow[t]{2}{*}{ Variables } & & \multicolumn{2}{|c|}{ Matched patients } & \multirow[t]{2}{*}{$\mathrm{p}$} \\
\hline & & Short-interval $(n=238)$ & Long-interval $(\mathrm{n}=238)$ & \\
\hline Gender ( M/F ) & & $225 / 13$ & $213 / 25$ & 0.062 \\
\hline Age, year & & $50(25-82)$ & $53(18-80)$ & 0.283 \\
\hline Hepatitis B ( \% ) & & $210(88.23 \%)$ & $201(84.45 \%)$ & 0.286 \\
\hline Hepatitis C ( \% ) & & $3(1.26 \%)$ & $8(3.436 \%)$ & 0.221 \\
\hline $\mathrm{WBC}, 10^{9} / \mathrm{L}$ & & $6.80(2.70-17.60)$ & $6.67(2.80-24.60)$ & 0.650 \\
\hline $\mathrm{RBC}, 10^{9} / \mathrm{L}$ & & $4.64(2.76-6.85)$ & $4.64(2.68-7.34)$ & 0.841 \\
\hline $\mathrm{HB}, \mathrm{g} / \mathrm{L}$ & & $138.30(83.70-192.00)$ & $137.00(65.40-185.00)$ & 0.328 \\
\hline PLT, $10^{9} / \mathrm{L}$ & & $199.40(31.00-749.70)$ & $188.85(12.10-604.00)$ & 0.598 \\
\hline $\mathrm{ALT}, \mathrm{U} / \mathrm{L}$ & & $43.70(8.70-322.70)$ & $46.4(9.1-455.00)$ & 0.805 \\
\hline AST, U/L & & $60.00(23.10-422.10)$ & $61.10(9.70-1332.50)$ & 0.758 \\
\hline GGT, U/L & & $178.15(24.50-1433.20)$ & $159.25(21.80$ - 1017.90) & 0.563 \\
\hline ALB, g/L & & $40.40(26.70-73.21)$ & $40.10(22.30-54.00)$ & 0.984 \\
\hline TBIL, $\mu \mathrm{mol} / \mathrm{L}$ & & $15.05(3.60-225.00)$ & $14.50(5.60-616.30)$ & 0.287 \\
\hline Creatine, $\mu \mathrm{mol} / \mathrm{L}$ & & $73.00(43.38-217.40)$ & $73.33(3.35-112.90)$ & 0.724 \\
\hline PT, sec & & $12.20(9.90-19.50)$ & $12.25(8.70-20.30)$ & 0.674 \\
\hline $\mathrm{CRP}, \mathrm{mg} / \mathrm{L}$ & & $11.73(0.25$ - 208.18) & 11.7 (0.46 - 241.3) & 0.682 \\
\hline INR & & $1.06(0.84-2.05)$ & $1.06(0.76-13.20)$ & 0.758 \\
\hline \multirow[t]{3}{*}{$\mathrm{AFP}, \mathrm{ng} / \mathrm{ml}$} & $<200$ & $86(36.14 \%)$ & $96(40.34 \%)$ & \\
\hline & $200-400$ & $8(3.36 \%)$ & $11(4.62 \%)$ & \\
\hline & $>400$ & $144(60.50 \%)$ & $131(55.04 \%)$ & 0.262 \\
\hline Tumor-Size, $\mathrm{cm}$ & & $10.0(1.0-19.1)$ & $9.7(1.0-20)$ & 0.940 \\
\hline
\end{tabular}




\begin{tabular}{|c|c|c|c|c|}
\hline \multirow[t]{2}{*}{ Variables } & & \multicolumn{2}{|c|}{ Matched patients } & \multirow[t]{2}{*}{$\mathrm{p}$} \\
\hline & & Short-interval (n= 238) & Long-interval $(\mathrm{n}=238)$ & \\
\hline \multirow[t]{2}{*}{ Tumor number } & Solitary & $86(36.13 \%)$ & $82(34.45 \%)$ & \\
\hline & Multifocal & $152(63.87 \%)$ & $156(65.55 \%)$ & 0.701 \\
\hline \multirow[t]{2}{*}{ PVTT } & Absent & $156(65.55 \%)$ & $163(68.49 \%)$ & \\
\hline & Present & $82(34.45 \%)$ & $75(31.51 \%)$ & 0.495 \\
\hline \multirow[t]{3}{*}{ Times of TACE } & 2 & $164(68.90 \%)$ & $146(61.35 \%)$ & \\
\hline & 3 & $49(20.59 \%)$ & $52(21.85 \%)$ & \\
\hline & $>=4$ & $25(10.51 \%)$ & $40(16.80 \%)$ & 0.051 \\
\hline Iodized oil, ml & & $18.9 \pm 7.4$ & $18.5 \pm 7.1$ & 0.625 \\
\hline \multirow[t]{2}{*}{ Child-Pugh stage } & A & $221(92.86 \%)$ & $223(93.70 \%)$ & \\
\hline & B & $17(7.14 \%)$ & $15(6.30 \%)$ & 0.714 \\
\hline \multirow[t]{3}{*}{ BCLC stage } & A & 48 (20.17\%) & $55(23.11 \%)$ & \\
\hline & B & $108(45.38 \%)$ & $107(44.96 \%)$ & \\
\hline & C & $82(34.45 \%)$ & $76(31.93 \%)$ & 0.423 \\
\hline \multirow[t]{3}{*}{ ABIL grade } & 1 & $133(55.88 \%)$ & $132(55.46 \%)$ & \\
\hline & 2 & $102(42.86 \%)$ & $104(43.70 \%)$ & \\
\hline & 3 & $3(1.26 \%)$ & $2(0.84 \%)$ & 0.960 \\
\hline
\end{tabular}

Values are presented as the median(Range) or $\mathrm{n}(\%)$

Abbreviations: WBC, white blood cell; RBC, red blood cell; HB, hemoglobin; PLT, platelet; ALT, alanine transaminase; AST, aspartate aminotransferase; GGT, gamma-glutamyl transpeptidase; ALB, albumin; TBIL, total bilirubin; PT, prothrombin time; CRP, C-reactive protein; INR, international normalized ratio; AFP, alpha fetoprotein; PVTT, portal vein tumor thrombus; TACE, transcatheter arterial chemoembolization; BCLC, Barcelona Clinical Liver Cancer; ABIL, Albumin-Bilirubin.
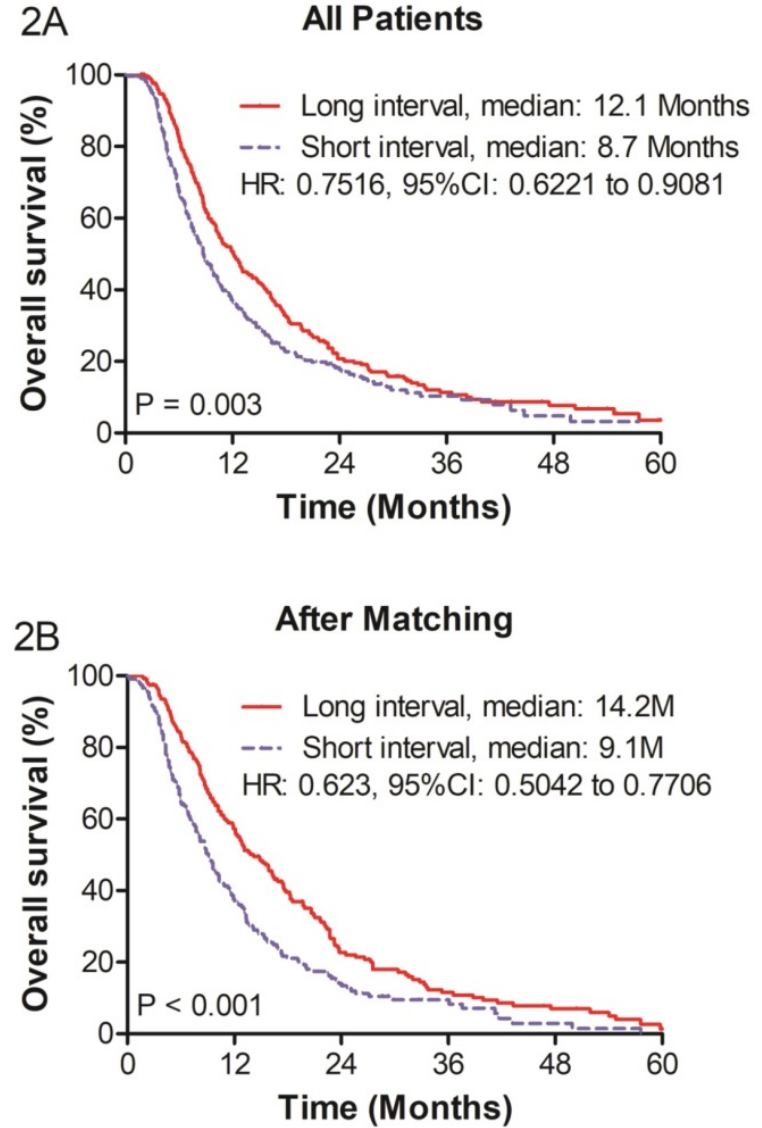

Figure 2. (A) Overall survival of before propensity score matching (PSM) and (B) overall survival of after PSM of patients with unrespectable HCC who underwent re-TACE.

\section{After PSM}

\section{Baseline}

We used PSM analysis to reduce the bias of covariances associated with patient selection between the SIG and LIG. The variables included in the analysis were gender, white blood cell count, gamma-glutamyl transpeptidase level, AFP, tumor size, and platelet count. In total, 238 patients in the SIG and 238 patients in the LIG were matched for further analyses. Any baseline characteristics that were significantly different between the patient groups were well-balanced after matching (Table 2).

The baseline biochemical results prior to TACE-2 were comparable between the SIG and the LIG. The GGT level was significantly higher (U/L, median: 201 vs. 190) in the SIG. The SIG had poorer liver function prior to TACE-2 than the LIG, a lower ALB level (g/L, $37.38 \pm 4.34$ vs. $38.82 \pm 5.78, \mathrm{P}=0.013)$, and more ALBI grade 2 and 3 patients $(64.3 \%$ vs. $54.6 \%, \mathrm{P}=0.027$; Supplementary Table 1).

The radiologic tumor responses after TACE-1 and TACE-2 are given in Supplementary Table 2. The SIG and LIG showed similar complete response (5.0\% vs. $7.6 \%)$, partial response $(37.8 \%$ vs. $37.0 \%)$, stable disease $(48.3 \%$ vs. $46.6 \%)$, and progressive disease $(8.8 \%$ vs. $8.8 \%)$ statuses after TACE-1 $(P=0.603)$. Moreover, no significant difference in tumor response after TACE-2 was observed between the SIG and LIG $(\mathrm{P}=0.852)$.

\section{Adverse events after TACE-2}

No statistically significant difference between the SIG and LIG was found for the overall number of patients who reported adverse events (Table 3; 29.4\% vs. $33.6 \%, \mathrm{P}=0.324)$. The most common treatment-related nonhematologic adverse events are listed in Table 3. The complications included hyperpyrexia ( $\mathrm{n}=42)$, vomiting $(\mathrm{n}=20)$, myelosuppression $(\mathrm{n}=1)$, hypohepatia $(\mathrm{n}=2)$, cholangitis $(\mathrm{n}=1)$, pulmonary infection $(\mathrm{n}=2)$, 
hepatapostema $(\mathrm{n}=1)$ and gastrointestinal bleeding $(\mathrm{n}=1)$ in the SIG. The complications included hyperpyrexia $(\mathrm{n}=55)$, vomiting $(\mathrm{n}=21)$, myelosuppression $(n=2)$ and hypohepatia $(n=2)$ in the LIG.

\section{Overall survival and prognostic factors}

In matched patients, the LIG had a significantly better OS than the SIG ( $\mathrm{P}<0.001$; HR, 0.623; 95\% CI, 0.504 to 0.771; Figure 3). The median OS rates of the SIG and LIG were 9.1 months and 14.2 months, respectively. The 1-, 2-, and 3-year survival rates were $37.5 \%, 17.1 \%$ and $9.9 \%$, respectively, in the SIG and $50.1 \%, 19.3 \%$ and $11.6 \%$ in the LIG, respectively.

Univariate analyses showed that a short interval $(\mathrm{P}=0.011)$, Child-Pugh $\mathrm{B}$ grade $(\mathrm{P}=0.016), \mathrm{AFP}>400$ $\mathrm{ng} / \mathrm{ml}(\mathrm{P}=0.027)$, PVTT presence $(\mathrm{P}=0.002)$, radiologic tumor response absent $(\mathrm{P}<0.001)$ and tumor size $>10 \mathrm{~cm}(\mathrm{P}=0.002)$ were significantly associated with a poor OS. Cox analysis identified a short interval $(\mathrm{HR}=1.399 ; 95 \% \mathrm{CI}: 1.135-1.725$; $\mathrm{P}=$ $0.002)$, radiologic tumor response absent $(\mathrm{HR}=0.636$; 95\% CI: $0.515-0.786$; P < 0.001), and tumor size $>10$ $\mathrm{cm}(\mathrm{HR}=1.366 ; 95 \% \mathrm{CI}: 1.105-1.675 ; \mathrm{P}=0.004)$ as independent adverse prognostic factors for OS (Table 4).
Table 3. Hematotoxicity and adverse event after TACE-2

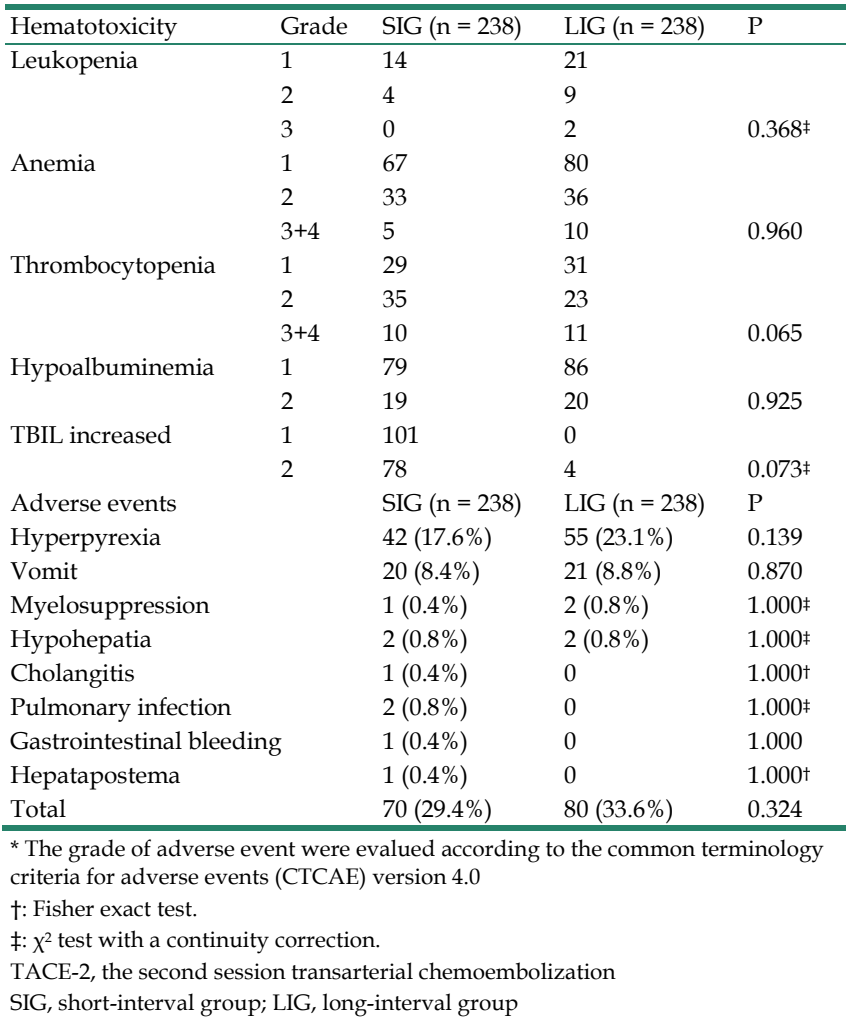

Table 4. Univariate and multivariate analysis for OS in matched patients

\begin{tabular}{|c|c|c|c|c|c|c|c|c|}
\hline \multirow[t]{3}{*}{ Variable } & \multirow[t]{3}{*}{ group } & \multicolumn{4}{|c|}{ Univariate analysis } & \multicolumn{3}{|c|}{ Multivariate analysis } \\
\hline & & \multicolumn{4}{|c|}{ OS (Months) } & \multirow[b]{2}{*}{ HR } & \multirow[b]{2}{*}{$95 \% \mathrm{CI}$} & \multirow[b]{2}{*}{$\mathrm{P}$} \\
\hline & & $\mathrm{n}=476$ & Median & $95 \% \mathrm{CI}$ & $\mathrm{P}$ & & & \\
\hline \multirow[t]{2}{*}{ Sex } & Male & 438 & 10.30 & $9.113-11.487$ & & & & \\
\hline & Female & 38 & 10.30 & $5.118-15.482$ & 0.977 & & & \\
\hline \multirow[t]{2}{*}{ Age (years) } & $<65$ & 417 & 9.90 & $8.904-10.896$ & & & & \\
\hline & $>=65$ & 59 & 14.20 & $11.934-16.466$ & 0.165 & & & \\
\hline \multirow[t]{2}{*}{ Child-Pugh stage } & A & 444 & 10.50 & $9.307-11.693$ & & & & \\
\hline & B & 32 & 7.00 & $2.605-11.395$ & 0.016 & & & \\
\hline \multirow[t]{3}{*}{ ALBI grade } & 1 & 193 & 11.80 & $10.083-13.517$ & & & & \\
\hline & 2 & 277 & 9.10 & $7.792-10.408$ & & & & \\
\hline & 3 & 6 & 8.80 & $1.599-16.001$ & 0.037 & & & \\
\hline \multirow[t]{3}{*}{ Alpha-Fetoprotein } & $<=200$ & 182 & 12.10 & 10.149 - 14.051 & & & & \\
\hline & $200-400$ & 19 & 17.30 & $1.00-35.024$ & & & & \\
\hline & $>400$ & 275 & 9.30 & $8.421-10.179$ & 0.027 & & & \\
\hline \multirow[t]{2}{*}{ Tumor number } & Unifocal & 168 & 9.80 & $7.905-11.695$ & & & & \\
\hline & Multifocal & 308 & 10.70 & $9.037-12.363$ & 0.622 & & & \\
\hline \multirow{2}{*}{ Portal vein tumor thrombus } & Present & 319 & 11.90 & $10.550-13.250$ & & & & \\
\hline & Absent & 157 & 8.30 & $7.331-9.269$ & 0.002 & & & \\
\hline \multirow[t]{2}{*}{ Albumin, g/L } & $<35$ & 73 & 9.30 & $7.915-10.685$ & & & & \\
\hline & $>=35$ & 403 & 10.50 & $9.270-11.730$ & 0.364 & & & \\
\hline \multirow[t]{2}{*}{ Total bilirubin, $\mu \mathrm{mol} / \mathrm{L}$} & $>17$ & 314 & 11.30 & $10.088-12.512$ & & & & \\
\hline & $<=17$ & 162 & 9.00 & $7.946-10.054$ & 0.208 & & & \\
\hline \multirow[t]{2}{*}{ Prothrombin time, sec. } & $>13.5$ & 412 & 10.30 & $8.999-11.601$ & & & & \\
\hline & $<=13.5$ & 64 & 10.20 & $5.813-14.587$ & 0.755 & & & \\
\hline \multirow[t]{2}{*}{ TACE interval } & $<=48$ & 238 & 8.90 & 7.779 - 10.021 & & & & \\
\hline & $>48$ & 238 & 12.10 & $10.445-13.755$ & 0.011 & 1.399 & $1.135-1.725$ & 0.002 \\
\hline \multirow[t]{2}{*}{ Radiologic tumor response } & Present & 271 & 13.00 & $11.438-14.562$ & & & & \\
\hline & Absent & 205 & 8.30 & $7.370-9.230$ & $<0.001$ & 0.636 & $0.515-0.786$ & $<0.001$ \\
\hline \multirow[t]{2}{*}{ Tumor size, cm } & $<=10$ & 251 & 12.40 & $10.714-14.086$ & & & & \\
\hline & $>10$ & 225 & 8.90 & $7.960-9.835$ & 0.002 & 1.366 & $1.105-1.675$ & 0.004 \\
\hline
\end{tabular}

OS: Overall survival; ALBI: Albumin-Bilirubin grade; TACE: transarterial chemoembolization; HR: hazard ratio; CI: Confidence Interval. 


\section{Outcomes within Barcelona Clinic liver cancer (BCLC) subgroups}

The median survivals of the SIG and LIG were 11.8 months and 12.8 months $(\mathrm{HR}=1.044 ; 95 \% \mathrm{CI}$ : 0.661 - 1.648; $\mathrm{P}=0.853$ ) for BCLC stage $\mathrm{A}$ and 10.9 months and 12.4 months for BCLC stage $\mathrm{B}$ (HR = 0.809; 95\% CI: $0.593-1.103 ; \mathrm{P}=0.180$ ), respectively. However, in the BCLC stage C group, the LIG $(n=76)$ had a better OS than the SIG $(\mathrm{n}=82)$, and the median survivals in the SIG and LIG were 5.8 months and 10.2 months, respectively $(\mathrm{HR}=0.525 ; 95 \% \mathrm{CI}: 0.359$ 0.767 ; P < 0.001). The 1-, 2-, and 3-year survival rates were $18.6 \%, 12.8 \%$, and $6.4 \%$ in the SIG and $44.3 \%$, $15.2 \%$, and $13.3 \%$ in the LIG, respectively (Figure 3 ).
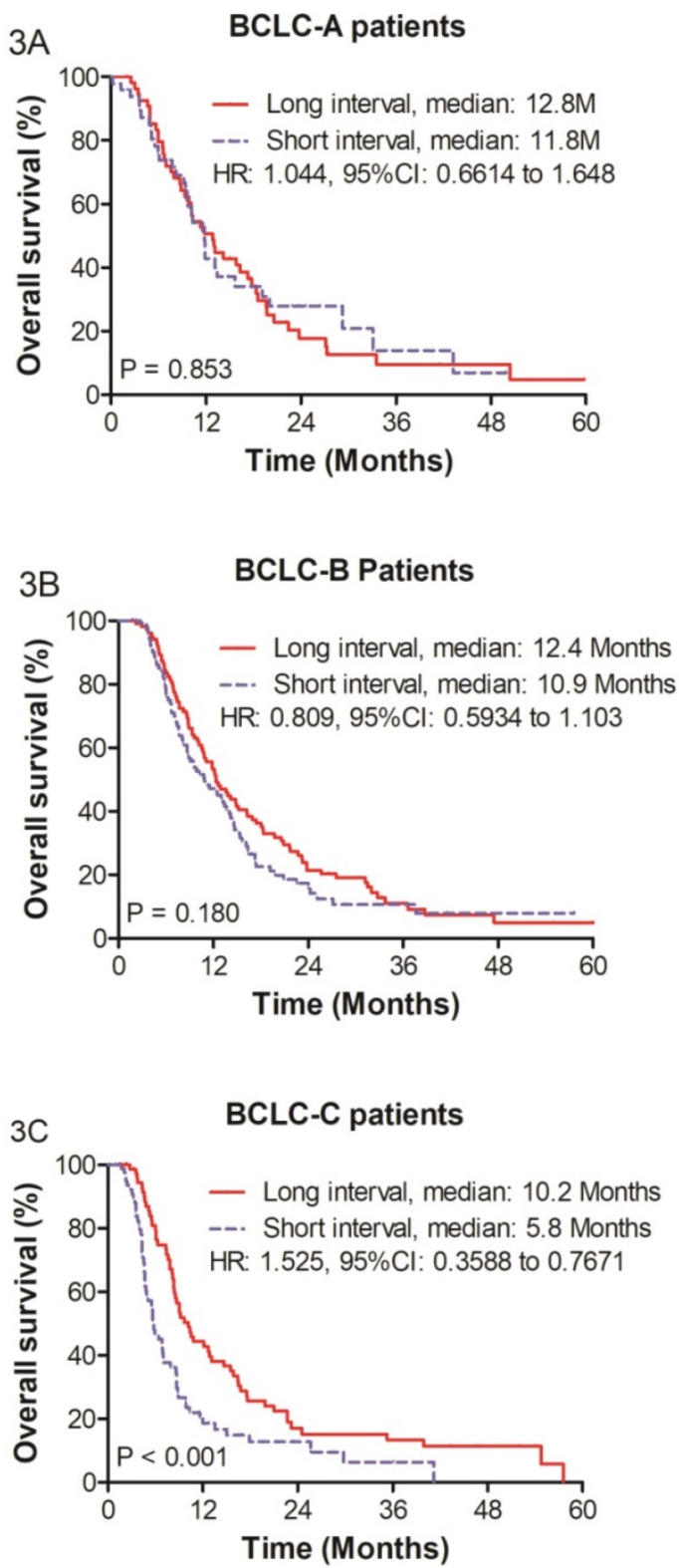

Figure 3. Survival analysis within Barcelona Clinic liver cancer (BCLC) subgroups: (A) Overall survival for BCLC-A subgroup patients (B) BCLC-B subgroup patients and (C) BCLC-C subgroup patients.

\section{Discussion}

Although TACE is the most widely used primary treatment for unresectable HCC, there is no standard TACE treatment schedule for patients with unresectable HCC and definitive data suggesting a superior strategy in terms of efficacy are lacking. In the current study, we compared outcomes between long- and short-interval TACE in patients with unresectable HCC. The OS was greater in the LIG than in the SIG before matching. After matching, we found that the LIG had a better OS than the SIG and that a short interval was an independent adverse prognostic factor for OS. Further analyses showed a significant difference in OS between the LIG and SIG among BCLC-C patients compared to BCLC-A and BCLC-B patients. Kim et al. reported that the TACE interval did not affect the survival outcomes of patients with Child-Pugh class A, but it did adversely affect survival in patients with Child-Pugh class B[20]. The difference between their study and ours may be due to the heterogeneity of the patient cohorts. On one hand, our study had more BCLC stage C patients who suffered from portal vein invasion (33.2\%, $158 / 476)$ than their study with a rate of $10.6 \%$ $(22 / 208)$. The significant difference in OS between the SIG and LIG in BCLC stage C patients may affect the results of all patients. On the other hand, the considerable number of cases in our study provides more convincing results by limiting any bias or confounding factors. Although our findings of this study cannot give definite TACE treatment schedule for unresectable HCC patients, our research has a positive significance to propose feasible suggestions for clinical practice. We can give difference interval of TACEs based on BCLC stages to increase the efficacy of treatment.

Most HCC patients who are candidates for TACE suffer from HBV infection, cirrhosis or major portal vein invasion, which are associated with a poor hepatic reserve[25]. These patients may experience more serious hepatic function damage after repeated TACE[26, 27]. Thus, a sufficient amount of time is necessary for recovery to normal hepatic function. This expectation was supported by a higher proportion of ALBI grade 1 patients in the LIG compared with the SIG (45.4\% vs. $35.7 \%, \mathrm{P}=0.027)$. Previous studies have shown that the ALBI grade is a prognostic factor for OS in patients with HCC [28, 29]. Therefore, we believe that a better OS is related to better liver function recovery after initial TACE as a result of a longer interval before the second session. Liver function in BCLC stage C patients who present portal vein invasion may be severely damaged by repeated TACE due to the original blood supply shortage. Therefore, these patients require more time 
to recover than BCLC-A and BCLC-B patients without PVTT. When planning for the next session TACE in BCLC-C patients, one should be cautious and extend the interval between sessions.

Additionally, the interval for repeated TACE in clinical practice ranges from one to three months or even longer in different medical centers. In China, the radiologic tumor response was generally evaluated within 3 to 6 weeks after initial TACE treatment. Therefore, most patients who are eligible for repeated TACE receive the next treatment session within 3 months. For patients with interval less than 21 days, the second TACE is a savage treatment for the failure of the first TACE treatment. A interval of repeated TACEs over 90 days suggests a poor compliance of treatment and follow-up. Therefore, we only included patients with TACE intervals of 21 to 90 days to avoid patient selection bias. The median TACE interval in this study was 48 days, which is consistent with results from other studies[18, 30]. We used 48 days as the cutoff to determine the long- and short-interval groups to adequately reflect real-life clinical practice.

Blocking the blood supply to a tumor by embolization and attacking tumor cells via cytotoxic chemotherapy contribute to the antitumoral effect of TACE[31]. Accordingly, the complications of TACE include postembolization syndrome, such as fever and abdominal pain, and side effects from chemotherapy, such as nausea, vomiting, deteriorated liver function and hematotoxicity[16, 32]. The side effects of TACE were frequent but mild and most cases of postembolization syndrome resolve within one month[33]. In this study, the median interval between the first two TACE sessions was 48 days; thus, most patients had recovered from the post-treatment side effects of TACE. Therefore, no differences in complications were found between the SIG and LIG.

Our study has some limitations. First, the potential bias of patient allocation cannot be completely avoided since this is a retrospective study. Second, we limited the interval range from 21 days to 90 days; the issue of whether longer or shorter intervals show similar findings remains uncertain. Such factors limit the generalizability of our conclusions and need to be resolved in the near future. Third, this is a single-center study; hence, these findings may not be consistent with results for other centers and geographic regions. To obtain more generalizable results, an independent external validation database may be necessary.

\section{Conclusions}

A long interval between the first two sessions of TACE resulted in a better OS than a short interval in
BCLC-C stage HCC patients.

\section{Supplementary Material}

Supplementary figures and tables. http://www.jcancer.org/v09p4000s1.pdf

\section{Abbreviations}

HCC, hepatocellular carcinoma; AFP, alpha-fetoprotein; $\mathrm{HBV}$, hepatitis $\mathrm{B}$ virus; $\mathrm{HCV}$, hepatitis $C$ virus; OS, overall survival; US, ultrasound; MVI, microvascular invasion; TACE, transarterial chemoembolization; ALB, albumin; TBIL, total bilirubin; ALT, alanine aminotransferase; AST, aspartate aminotransferase; WBC, white blood cell; $\mathrm{RBC}$, red blood cell; PLT, platelet; PT, prothrombin time; INR, international normalized ratio; ALBI, Albumin-Bilirubin grade; BCLC, Barcelona clinic liver cancer stage; mRECIST, The modified response evaluation criteria in solid tumors criterion; SIG, short-interval group; LIG, long-interval group; PSM, propensity score matching; PVTT, portal vein tumor thrombus; HR, hazard ratio; CI, Confidence Interval.

\section{Acknowledgements}

This manuscript is an original research. This paper was not presented at an RSNA meeting or a future meeting. This work was supported by grants from the National Natural Science Foundation of China (No. 81372571), the State "973 Program" of China (No. 2014CB542005), the Sun Yat-Sen University Clinical Research 5010 Program (No. 2012010) and the Fundamental Research Funds for the Central Universities (No. 17ykzd34).

The study protocol conformed to the ethical guidelines of the 1975 Declaration of Helsinki, and the Ethics Committee of the Sun Yat-Sen University Cancer Center approved this study. Written informed consent was obtained before treatment.

\section{Availability of data and materials}

All data generated or analysed during this study are included in this published article and its supplementary information files.

\section{Author Contributions}

Zhiwen Yang performed the study design, the statistical analysis, interpreted the results and drafted the manuscript. Wei He contributed to the statistical analysis, interpreted the results and drafted the manuscript. Yun Zheng drafted and revised the manuscript. Ruhai Zou contributed to study design and intellectual content. Wenwu liu and Yuanping Zhang helped to perform the statistical analysis and interpreted the data. Chenwei Wang, Yongjin Wang and Yichuan Yuan acquired the data. Binkui Li 
contributed to study design and critically revised the manuscript. Yunfei Yuan conceived of the study, critically revised and approved the manuscript.

\section{Competing Interests}

\section{All authors declare no conflict of interests.}

\section{References}

1. Torre LA, Bray F, Siegel RL, Ferlay J, Lortet-Tieulent J, Jemal A. Global cancer statistics, 2012. CA: a cancer journal for clinicians. 2015;65(2):87-108.

2. Lencioni R, de Baere T, Soulen MC, Rilling WS, Geschwind JF. Lipiodol transarterial chemoembolization for hepatocellular carcinoma: A systematic review of efficacy and safety data. Hepatology. 2016;64(1):106-16.

3. Forner A, Llovet JM, Bruix J. Chemoembolization for intermediate HCC: is there proof of survival benefit? J Hepatol. 2012;56(4):984-6.

4. Luo J, Guo RP, Lai EC, Zhang YJ, Lau WY, Chen MS, et al. Transarterial chemoembolization for unresectable hepatocellular carcinoma with portal vein tumor thrombosis: a prospective comparative study. Ann Surg Oncol. 2011;18(2):413-20.

5. Takayasu K, Arii S, Ikai I, Omata M, Okita K, Ichida T, et al. Prospective cohort study of transarterial chemoembolization for unresectable hepatocellular carcinoma in 8510 patients. Gastroenterology. 2006;131(2):461-9.

6. Wang JH, Changchien CS, Hu TH, Lee CM, Kee KM, Lin CY, et al. The efficacy of treatment schedules according to Barcelona Clinic Liver Cancer staging for hepatocellular carcinoma - Survival analysis of 3892 patients. European Journal of Cancer. 2008;44(7):1000-6.

7. Lewandowski RJ, Mulcahy MF, Kulik LM, Riaz A, Ryu RK, Baker TB, et al. Chemoembolization for hepatocellular carcinoma: comprehensive imaging and survival analysis in a 172-patient cohort. Radiology. 2010;255(3):955-65

8. Camma C, Schepis F, Orlando A, Albanese M, Shahied L, Trevisani F, et al. Transarterial chemoembolization for unresectable hepatocellular carcinoma: meta-analysis of randomized controlled trials. Radiology. 2002;224(1):47-54

9. Llovet JM, Real MI, Montana X, Planas R, Coll S, Aponte J, et al. Arterial embolisation or chemoembolisation versus symptomatic treatment in patients with unresectable hepatocellular carcinoma: a randomised controlled trial. Lancet. 2002;359(9319):1734-9.

10. Georgiades C, Geschwind J-F, Harrison N, Hines-Peralta A, Liapi E, Hong K, et al. Lack of response after initial chemoembolization for hepatocellular carcinoma: does it predict failure of subsequent treatment? Radiology. 2012;265(1):115-23

11. Kim KM, Kim JH, Park IS, Ko GY, Yoon HK, Sung KB, et al. Reappraisal of repeated transarterial chemoembolization in the treatment of hepatocellular carcinoma with portal vein invasion. J Gastroenterol Hepatol. 2009;24(5):806-14.

12. Xia J, Ren Z, Ye S, Sharma D, Lin Z, Gan Y, et al. Study of severe and rare complications of transarterial chemoembolization (TACE) for liver cancer. Eur J Radiol. 2006;59(3):407-12.

13. Buijs M, Vossen JA, Frangakis C, Hong K, Georgiades CS, Chen Y, et al Nonresectable hepatocellular carcinoma: long-term toxicity in patients treated with transarterial chemoembolization--single-center experience. Radiology. 2008;249(1):346-54

14. Chan AO, Yuen MF, Hui CK, Tso WK, Lai CL. A prospective study regarding the complications of transcatheter intraarterial lipiodol chemoembolization in patients with hepatocellular carcinoma. Cancer. 2002;94(6):1747-52.

15. Moreno-Luna LE, Yang JD, Sanchez W, Paz-Fumagalli R, Harnois DM, Mettler $\mathrm{TA}$, et al. Efficacy and safety of transarterial radioembolization versus chemoembolization in patients with hepatocellular carcinoma. Cardiovasc Intervent Radiol. 2013;36(3):714-23

16. Clark TW. Complications of hepatic chemoembolization. Semin Intervent Radiol. 2006;23(2):119-25

17. Adhoute X, Penaranda G, Naude S, Raoul JL, Perrier H, Bayle O, et al. Retreatment with TACE: the ABCR SCORE, an aid to the decision-making process. J Hepatol. 2015;62(4):855-62.

18. Sieghart W, Hucke F, Pinter M, Graziadei I, Vogel W, Muller C, et al. The ART of decision making: retreatment with transarterial chemoembolization in patients with hepatocellular carcinoma. Hepatology. 2013;57(6):2261-73.

19. Arizumi T, Ueshima K, Iwanishi M, Minami T, Chishina H, Kono M, et al. Evaluation of ART Scores for Repeated Transarterial Chemoembolization in Japanese Patients with Hepatocellular Carcinoma. Oncology. 2015;89 Suppl 2:4-10.

20. Kim H-D, An J, Kim JH, Gwon DI, Shin JH, Ko G-Y, et al. Impact of the Interval between Transarterial Chemoembolization Sessions on Survival in Patients with Unresectable Hepatocellular Carcinoma. Journal of Vascular and Interventional Radiology. 2016;27(4):504-13.

21. European Association For The Study Of The L, European Organisation For R, Treatment Of C. EASL-EORTC clinical practice guidelines: management of hepatocellular carcinoma. J Hepatol. 2012;56(4):908-43.

22. Peng ZW, Zhang YJ, Liang HH, Lin XJ, Guo RP, Chen MS. Recurrent hepatocellular carcinoma treated with sequential transcatheter arterial chemoembolization and RF ablation versus RF ablation alone: a prospective randomized trial. Radiology. 2012;262(2):689-700.

23. Lencioni R, Llovet JM. Modified RECIST (mRECIST) assessment for hepatocellular carcinoma. Semin Liver Dis. 2010;30(1):52-60.

24. Chen AP, Setser A, Anadkat MJ, Cotliar J, Olsen EA, Garden BC, et al. Grading dermatologic adverse events of cancer treatments: the Common Terminology Criteria for Adverse Events Version 4.0. Journal of the American Academy of Dermatology. 2012;67(5):1025-39.

25. Chen W, Zheng R, Baade PD, Zhang S, Zeng H, Bray F, et al. Cancer statistics in China, 2015. CA: a cancer journal for clinicians. 2016;66(2):115-32.

26. Jang JW, Choi JY, Bae SH, Kim CW, Yoon SK, Cho SH, et al. Transarterial chemo-lipiodolization can reactivate hepatitis B virus replication in patients with hepatocellular carcinoma. J Hepatol. 2004;41(3):427-35

27. Jeon SH, Park KS, Kim YH, Shin YS, Kang MK, Jang BK, et al. [Incidence and risk factors of acute hepatic failure after transcatheter arterial chemoembolization for hepatocellular carcinoma]. Korean J Gastroenterol. 2007;50(3):176-82.

28. Xu L, Peng ZW, Chen MS, Shi M, Zhang YJ, Guo RP, et al. Prognostic nomogram for patients with unresectable hepatocellular carcinoma after transcatheter arterial chemoembolization. J Hepatol. 2015;63(1):122-30.

29. Johnson PJ, Berhane S, Kagebayashi C, Satomura S, Teng M, Reeves HL, et al. Assessment of liver function in patients with hepatocellular carcinoma: a new evidence-based approach-the ALBI grade. Journal of Clinical Oncology. 2014;33(6):550-8

30. Tang Q-h, Li A-j, Yang G-m, Lai EC, Zhou W-p, Jiang Z-h, et al. Surgical resection versus conformal radiotherapy combined with TACE for resectable hepatocellular carcinoma with portal vein tumor thrombus: a comparative study. World journal of surgery. 2013;37(6):1362-70

31. Huppert P. Current concepts in transarterial chemoembolization of hepatocellular carcinoma. Abdominal imaging. 2011;36(6):677-83.

32. Tarazov P, Polysalov V, Prozorovskij K, Grishchenkova I, Rozengauz E. Ischemic complications of transcatheter arterial chemoembolization in liver malignancies. Acta Radiologica. 2000;41(2):156-60.

33. Vogl TJ, Lammer J, Lencioni R, Malagari K, Watkinson A, Pilleul F, et al. Liver, gastrointestinal, and cardiac toxicity in intermediate hepatocellular carcinoma treated with PRECISION TACE with drug-eluting beads: results from the PRECISION V randomized trial. AJR Am J Roentgenol. 2011;197(4):W562-70. 